\title{
QCD Sum Rules Calculation of Heavy $\Lambda$ Semileptonic Decay
}

\author{
H.G. Dosch ${ }^{1}$, E. Ferreira ${ }^{2}$, M. Nielsen ${ }^{3}$ and R. Rosenfeld ${ }^{4}$ \\ ${ }^{1}$ Institut für Theoretische Physik, Universität Heidelberg \\ Philosophenweg 16, D-6900 Heidelberg, Germany \\ ${ }^{2}$ Instituto de Física, Universidade Federal do Rio de Janeiro \\ C.P. 68528, Rio de Janeiro 21945-970, RJ, Brazil \\ ${ }^{3}$ Instituto de Física, Universidade de São Paulo \\ C.P. 66318, 05315-970 São Paulo, SP, Brazil \\ ${ }^{4}$ Instituto de Física Teórica, Universidade Estadual Paulista \\ Rua Pamplona, 145; 01405-900 - São Paulo,SP, Brazil
}

\begin{abstract}
We set up sum rules for heavy lambda decays in a full QCD calculation which in the heavy quark mass limit incorporates the symmetries of heavy quark effective theory. For the semileptonic $\Lambda_{c}$ decay we obtain a reasonable agreement with experiment. For the $\Lambda_{b}$ semileptonic decay we find at the zero recoil point a violation of the heavy quark symmetry of about $20 \%$.
\end{abstract}

PACS Numbers : 12.38.Lg, 13.30.Ce, 14.65.Dw , 14.65.Fy 
The semileptonic decays of hadrons are the most valuable source of information for the determination of the Cabbibo-Kobayashi-Maskawa (CKM) matrix elements, but unfortunately these fundamental quantities of the standard model have to be disentangled from the effects of strong interactions occurring inside the hadrons. In the limit where the initial and final quarks are infinitely heavy the degrees of freedom can be integrated out and the remaining heavy quark effective theory (HQET) ( [1 4, for a review see [5]) can make a number of rigorous statements that are independent of the details of the strong interaction. However, the corrections to the heavy quark effective theory due to finite quark masses are by no means negligible and therefore one has to look for more realistic treatments of the effects of strong interactions on the semileptonic decays. QCD sum rules [6] (for reviews see [4,8]) are one of the most widely used and best founded approaches for these purposes, treating non-perturbative effects analytically with a limited input of phenomenological parameters, with good success in the calculation of corrections to HQET (see [5] and references therein). Particularly, QCD sum rules have shown that the non-leading corrections to HQET can be quite appreciable, even for hadrons with $b$-quarks [9]:10].

Semileptonic decays of charmed into strange hadrons are among the best investigated processes of this kind. The relevant CKM matrix element $V_{c s}$ is well determined, so that calculations of these decays may provide very good tests of the applied method. On the other hand, there are quite serious discrepancies between experiments and HQET in the ratios of the lifetimes of beautiful baryons to beautiful mesons (see e.g. [11]), and hence it is of prime interest to investigate all decay channels of the $\Lambda_{b}$ baryon.

In this letter we evaluate the semileptonic decays of heavy $\Lambda$-baryons in the QCD sum rule approach as was developed for heavy meson decays in reference [12]. This approach treats full QCD but also reproduces the symmetries of HQET. It is therefore a very interesting method for the investigation of deviations from HQET in the decay $\Lambda_{b} \rightarrow \Lambda_{c} \ell \nu_{\ell}$.

We label generically the initial channel by $I$ and the final channel by $F$. For the decay $\Lambda_{I} \rightarrow \Lambda_{F}+\ell+\nu_{\ell}$ we start from the three-point function of the weak transition current from a initial to a final quark $J_{\mu}=\bar{Q}_{F} \gamma_{\mu}\left(1-\gamma_{5}\right) Q_{I}$ and the interpolating fields of the initial and 
final baryons $\eta_{\Lambda_{I}}$ and $\eta_{\Lambda_{F}}$

$$
\Pi_{\mu}\left(p_{F}, p_{I}\right)=(i)^{2} \int d^{4} x d^{4} y\left\langle 0\left|T\left\{\eta_{\Lambda_{F}}(x) J_{\mu}(0) \bar{\eta}_{\Lambda_{I}}(y)\right\}\right| 0\right\rangle e^{i p_{F} x} e^{-i p_{I} y} .
$$

The experimental information is contained in the decay amplitude [13]

$$
\begin{aligned}
& \left\langle\Lambda_{F}\left(p_{F}\right)\left|J_{\mu}\right| \Lambda_{I}\left(p_{I}\right)\right\rangle= \\
& \quad \bar{u}\left(p_{F}\right)\left[\gamma_{\mu}\left(F_{1}^{V}+F_{1}^{A} \gamma_{5}\right)+i \sigma_{\mu \nu} q^{\nu}\left(F_{2}^{V}+F_{2}^{A} \gamma_{5}\right)+q_{\mu}\left(F_{3}^{V}+F_{3}^{A} \gamma_{5}\right)\right] u\left(p_{I}\right),
\end{aligned}
$$

where $q=p_{F}-p_{I}$ and the form factors are functions of $t=q^{2}$.

We relate the theoretical (Eq. (11)) with the physical (Eq. (2)) quantities by inserting intermediate states into Eq. (1), which is then evaluated in the not so deep Euclidean region where $p_{F}^{2}<M_{\Lambda_{F}}^{2}$ and $p_{I}^{2}<M_{\Lambda_{I}}^{2}$.

We introduce the couplings $f_{F_{1}}$ and $f_{I_{1}}$ of the currents with the respective hadronic states

$$
\begin{aligned}
\left\langle 0\left|\eta_{\Lambda_{F}}\right| \Lambda\left(p_{F}\right)\right\rangle & =f_{F_{1}} u\left(p_{F}\right) \\
\left\langle\Lambda_{I}\left(p_{I}\right)\left|\bar{\eta}_{\Lambda_{I}}\right| 0\right\rangle & =f_{I_{1}} \bar{u}\left(p_{I}\right),
\end{aligned}
$$

and obtain the phenomenological representation of Eq. (ID)

$$
\begin{aligned}
\Pi_{\mu}^{(\text {phen })}\left(p_{F}, p_{I}\right) & =\frac{\left(f_{F_{1}} p_{F}+f_{F_{2}}\right)}{p_{F}^{2}-M_{\Lambda_{F}}^{2}}\left[\gamma_{\mu}\left(F_{1}^{V}+F_{1}^{A} \gamma_{5}\right)+i \sigma_{\mu \nu} q^{\nu}\left(F_{2}^{V}+F_{2}^{A} \gamma_{5}\right)\right. \\
& \left.+q_{\mu}\left(F_{3}^{V}+F_{3}^{A} \gamma_{5}\right)\right] \frac{\left(f_{I_{1}} p_{I}+f_{I_{2}}\right)}{p_{I}^{2}-M_{\Lambda_{I}}^{2}}+\text { higher resonances }
\end{aligned}
$$

where we have defined $f_{F_{2}}=f_{F_{1}} M_{\Lambda_{F}}$ and $f_{I_{2}}=f_{I_{1}} M_{\Lambda_{I}}$.

The theoretical expression is evaluated by performing the operator product expansion of the operator in Eq. (11) and then taking the expectation value with respect to the physical vacuum. The term from the unit operator gives the usual perturbative contribution, while the vacuum expectation values of the other operators in the expansion give the nonperturbative corrections proportional to the condensates of the respective operators. Thus

$$
\Pi_{\mu}^{\text {theor }}=\Pi_{\mu}^{\text {pert }}+\sum_{i} \Pi_{\mu}^{\text {nonpert }(i)}
$$


where the index i refers to the dimensions of the condensates.

As usual we evaluate the form factors $F_{a}^{V, A}$ of Eq. (2) $(a=1,2,3)$ by matching the phenomenological representation Eq. (5) of the three point function with the theoretical counterpart in Eq. (6). We project out sum rules for the products $F_{a}^{V, A}\left(q^{2}\right) f_{I_{i}} f_{F_{k}} \quad(i, k=$ 1,2) of the invariant amplitudes in Eq. (2) and the current couplings $f$ of Eqs. (3), (4), by performing appropriate traces of Eq. (5). We thus obtain four sum rules for each amplitude $F_{a}^{V, A}(t)$, but we use only those based on $f_{I_{1}}$ and $f_{F_{1}}$ since for them the imaginary part is positive definite. After this projection has been performed the treatment follows very closely the lines given in ref. [12]. We make the usual assumption that the contributions of the higher resonances (and the continuum) can be adequately approximated by the perturbative contributions above certain thresholds $s=p_{I}^{2} \geq s_{0}$ and $u=p_{F}^{2} \geq u_{0}$ and we use the Borel improvement by performing a double Laplace transform of the spectral functions of the theoretical expressions. A crucial ingredient for the incorporation of the HQET symmetries is to express the current couplings $f_{I_{i}}$ and $f_{F_{k}}$ also through QCD sum rules and relate the Borel parameters in the same way as explained in reference [12]. This also leads to a considerable reduction of the sensitivity to input parameters, like the continuum thresholds $s_{0}$ and $u_{0}$, and to radiative corrections [14].

As it is well known from two-point sum rules for baryons [15 17], there is a continuum of choices for the interpolating currents. Of course the results should in principle be independent of the choice of the current (except for pathological cases which couple very weakly to the ground state), but the justification of the approximations depends on the choice made. In this letter we concentrate on a very simple interpolating current where the two light quarks form a singlet spin and isospin state, namely

$$
\eta_{\Lambda_{Q}}=\epsilon_{A B C}\left(\bar{u}^{A} \gamma^{5} d^{B}\right) Q^{C}
$$

where $u^{A}$ and $d^{B}$ stands for the Dirac field of light quarks of colours $A$ and $B$, and $Q^{C}$ represents a heavy quark ( $b$ or $c$ ) of color $C$. This current couples strongly to the $\Lambda$ states in the heavy quark limit. With this choice of current the quark condensate and the mixed 
gluon-quark condensate do not contribute to Eq.(6). The gluon condensate does contribute, but experience with baryonic two-point functions and mesonic three point functions teaches us that it is of little influence. We are thus left with only the perturbative and the four quark condensate contributions.

In order to estimate the four quark condensate we use the factorization

$$
\left\langle\bar{d}_{\alpha}^{A} \bar{u}_{\beta}^{B} u_{\beta^{\prime}}^{B^{\prime}} d_{\alpha^{\prime}}^{A^{\prime}}\right\rangle=\frac{\kappa}{12^{2}} \delta_{\beta \beta^{\prime}} \delta_{\alpha \alpha^{\prime}} \delta^{A A^{\prime}} \delta^{B B^{\prime \prime}}\langle\bar{u} u\rangle\langle\bar{d} d\rangle
$$

where the parameter $\kappa$, which ranges from 1 to 3 , represents the deviation from the factorization hypothesis [7]. For the value of the quark condensate we take $<q \bar{q}>=-(230 \mathrm{MeV})^{3}$.

As general results we obtain

$$
F_{1}^{A}(t)=-F_{1}^{V}(t) ; F_{2}^{V}(t)=F_{3}^{V}(t)=F_{2}^{A}(t)=F_{3}^{A}(t)=0 .
$$

In Fig. 1 we show the behaviour of the contributions to the form factor $F_{1}^{V}$ at $t=0$ for the process $\Lambda_{c} \rightarrow \Lambda \ell \nu_{\ell}$ for $\kappa=1$ as function of the Borel mass $M_{F}^{2}$. We have used $M_{\Lambda_{c}}=2.285$ $\mathrm{GeV}, M_{\Lambda}=1.115 \mathrm{GeV}, m_{c}=1.4 \mathrm{GeV}, m_{s}=0.17 \mathrm{GeV}$ and $V_{c s}=0.975$. We observe that the contributions from the continuum and from the four quark condensate are comparable and tend to stabilize each other for values $M_{F}^{2} \geq 5 \mathrm{GeV}^{2}$. This seems to be a rather large value for the Borel mass. However, the influence of the 6-dimensional condensate is still large at that value and we expect the contributions of higher dimensional condensates to be very important at smaller Borel masses. A classical sum rule window where the perturbative and non-perturbative contributions are in equilibrium is thus the range above $5 \mathrm{GeV}^{2}$. In that range we obtain $F_{1}^{V}(0)=0.51 \pm 0.02$ for $\kappa=1$. We have also calculated the $t$ - dependence of this form factor in the range $0 \leq t \leq 0.6 \mathrm{GeV}^{2}$ where we do not encounter difficulties with non-Landau singularities [12]. The $t$-dependence is represented with dots in Fig. 2 for two different choices of the four quark condensate $(\kappa=1,2)$. It can be very well approximated by a pole fit (solid line for $\kappa=2$ and dashed line for $\kappa=1$ ). Using this form factor we obtain for the width

$$
\Gamma\left(\Lambda_{c} \rightarrow \Lambda+e^{+}+\nu_{e}\right)=(1.0 \pm 0.3) \times 10^{-13} \mathrm{GeV}
$$


Within the errors this value agrees with the reported experimental value [22]

$$
\Gamma\left(\Lambda_{c} \rightarrow \Lambda+e^{+}+\nu_{e}\right)=(0.74 \pm 0.15) \times 10^{-13} \mathrm{GeV}
$$

The values in Eq. (9) yield an asymmetry parameter $\alpha=-1$ whereas the observed value [22 is $-0.82 \pm 0.10$.

The overwhelming source of the theoretical error is the uncertainty in the value of the four quark condensate. Variations of the quark masses and continuum thresholds within the reasonable limits $0.13 \leq m_{s} \leq 0.17 \mathrm{GeV}, 1.25 \leq m_{c} \leq 1.45 \mathrm{GeV}, 7.8 \leq s_{0} \leq 8.9 \mathrm{GeV}^{2}$ and $2.6 \leq u_{0} \leq 3.4 \mathrm{GeV}^{2}$, are negligible as compared to the errors introduced by the variation of the four quark condensate.

Considering $\kappa$ in the range $1 \leq \kappa \leq 3$ the form factor at the zero recoil point $t_{\max }=$ $\left(M_{\Lambda_{c}}-M_{\Lambda}\right)^{2}$ is $F_{1}^{V}\left(t_{\max }\right)=0.79 \pm 0.07$, and is less dependent on the input parameters than for smaller values of $t$.

The form factors and the decay width of the semileptonic $\Lambda_{b}$ decay was calculated in the same way. We have used $M_{\Lambda_{b}}=5.65 \mathrm{GeV}, M_{\Lambda_{c}}=2.285 \mathrm{GeV}, m_{b}=4.6 \mathrm{GeV}, m_{c}=1.4$ $\mathrm{GeV}$ and $V_{c b}=0.04$.

The behaviour of the OPE contributions to the form factor $F_{1}^{V}$ at $t=0$ for the process $\Lambda_{b} \rightarrow \Lambda_{c} \ell \nu_{\ell}$ as function of the Borel mass $M_{F}^{2}$ is shown in Fig. 3 for $\kappa=1$. Here the relative importance of the four quark condensate is smaller than in the $\Lambda_{c}$ decay. In this case the region in the Borel mass where the perturbative and non-perturbative contributions are in equilibrium is above $10 \mathrm{GeV}^{2}$ and we obtain $F_{1}^{V}(0)=0.36 \pm 0.02$ for $\kappa=1$. The $t$-dependence of this form factor can be calculated in the range $0 \leq t \leq 8 \mathrm{GeV}^{2}$ (which covers the major part of the kinematically allowed region $0 \leq t \leq 11.34 \mathrm{GeV}^{2}$ ) without encountering difficulties with non-Landau singularities [12]. The $t$-dependence is again well approximated by pole fits, as can be seen in Fig. 4. The extrapolation of the fits to the maximal momentum transfer value, $t_{\max }=\left(m_{\Lambda_{b}}-m_{\Lambda_{c}}\right)^{2}$, yields $F_{1}^{V}=-F_{1}^{A}=0.74$ for $\kappa=1$ or 2 . This value is remarkably stable against variations of the input parameters like $\kappa, s_{0}, u_{0}$ and the Borel mass. In the interval $1 \leq \kappa \leq 3$ we estimate the errors to be 


$$
F_{1}^{V}\left(t_{\max }\right)=-F_{1}^{A}\left(t_{\max }\right)=0.76 \pm 0.05 .
$$

In HQET this value is just the Isgur-Wise function at zero quark recoil and is predicted to be 1 . We thus find a strong deviation of the heavy quark symmetry in the semileptonic decay $\Lambda_{b} \rightarrow \Lambda_{c}$. The deviation is larger than in the $B \rightarrow D$ decays, where the corresponding value is about 0.9 , in accordance with sum rules in full QCD [18], and corrections to HQET [19]. We have checked that this strong deviation is almost entirely due to the small mass of the charmed quark. We have also calculated the semileptonic decay rate, which turns out to be much more sensitive to the input parameters than the value at the zero recoil amplitude. We have obtained for the decay width

$$
\Gamma\left(\Lambda_{b} \rightarrow \Lambda_{c}+e+\bar{\nu}_{e}\right)=(1.8 \pm 0.3) \times 10^{-14} \mathrm{GeV},
$$

where the errors reflect variations of $\kappa$ from 1 to 3 and of the other parameters within reasonable limits. This value is consideraby smaller than other predictions [13,20,21], which range from 3.5 to $6 \times 10^{-14} \mathrm{GeV}$. It is interesting to note that the $1 / m_{Q}$ corrections to HQET tend to increase the width [13,21], whereas our result clearly indicates that the width in full QCD is smaller than in HQET. The decay width obtained by us stays well below the experimental upper limit [22] given by $\Gamma\left(\Lambda_{b} \rightarrow \Lambda_{c}^{+} \ell^{-} \bar{\nu}_{\ell}+\right.$ anything $)=(5.8 \pm 0.2) \times 10^{-14}$ $\mathrm{GeV}$.

Important violations of HQET for some form factors are not unplausible due to the large difference between the mass of the $\Lambda_{b}$ and the $b$-quark ( see ref. [5], table 4.1), but such a large deviation for Luke protected form factors like $F_{1}^{A}\left(t_{\max }\right)$ is certainly not expected. Although we can check that our treatment repects HQET we cannot, in the approach adopted here, evaluate reliably the $1 / m_{Q}$ and $1 / m_{Q}^{2}$ corrections. We can only state that a full QCD calculation which respects HQET and gives reasonable results for the experimentally known semileptonic decay of the $\Lambda_{c}$ indicates a large violation of heavy quark effective symmetry.

A detailed description of our procedure and the application to other decays and observables, as well as the dependence of the results with the interpolating fields, will be given in a forthcoming publication [23]. 
Acknowledgements We thank L.A. Barreiro for discussions. Financial support from CNPq, USP, FAPESP, FAPERJ (Brazil) and DAAD (Germany) is gratefully acknowledged. 


\section{REFERENCES}

[1] M.B. Voloshin and M.A. Shifman, Sov. J. Nucl. Phys. 45, 292 (1987); 47,511 (1988).

[2] S. Nussinov and W. Wetzel, Phys. Rev D 36, 130 (1987).

[3] N. Isgur and M.B. Wise, Phys. Lett. B237, 527 (1990); Nucl. Phys. B348, 276 (1991).

[4] H. Georgi, Phys. Lett. B240, 447 (1990); Nucl. Phys. B348, 293 (1991).

[5] M. Neubert, Phys. Rep. 245, 259 (1994).

[6] M.A. Shifman, A.I. Vainshtein and V.I. Zakharov, Nucl. Phys. B147, 385 (1979); B147, 448 (1979).

[7] S. Narison, QCD Spectral Sum Rules, World Scientific, Singapore 1989.

[8] M.A. Shifman, Vacuum Structure and QCD Sum Rules, North Holland, Amsterdam 1992.

[9] E. Bagan, P. Ball, V.M. Braun and H.G. Dosch, Phys. Lett. B278, 457 (1992).

[10] S. Narison, Z.Phys. C55, 671, (1992).

[11] M. Neubert, Theory of Beauty Lifetimes, Second International Conference on B Physics and CP Violation, Honolulu 1997, hep-ph/9707217.

[12] P. Ball, V.M. Braun and H.G. Dosch, Phys. Rev. D44, 3567 (1991).

[13] J.G. Körner, M. Krämer and D. Pirjol, Prog. Part. Nucl. Phys. 33 , 787 (1994).

[14] E. Bagan, P. Ball and P. Gosdzinsky, Phys. Lett B301, 249 (1993).

[15] B.L. Ioffe, Nucl. Phys. B188, 317 (1981); Erratum: B191, 591 (1981).

[16] Y. Chung, et al. Nucl. Phys. B197, 55 (1982).

[17] E. Bagan, M. Chabab, H.G. Dosch and S. Narison, Phys. Lett. B278, 367 (1992); B287, 176 (1992); B301, 243 (1993). 
[18] P. Ball, Phys. Lett. B281, 133 (1992).

[19] M. Neubert, Phys. Lett. B338, 84 (1994).

[20] M. A. Ivanov, V. E. Lyubovitskij, J. G. Körner and P. Kroll, Phys. Rev. D56, 348 (1997).

[21] Y. Dai, C. Huang, M. Huang and C. Liu, Phys. Lett. B387, 379 (1996).

[22] Particle Data Group, Phys. Rev. D54, 1 (1996).

[23] H.G. Dosch, E. Ferreira, M. Nielsen and R. Rosenfeld, in preparation. 


\section{FIGURES}

FIG. 1. The sum rule values for the decay amplitude $F_{1}^{V}$ at $t=0$ for the process $\Lambda_{c} \rightarrow \Lambda \ell \nu_{\ell}$ as function of the Borel mass $M_{F}^{2}$. The long-dashed line is the perturbative contribution, the short-dashed line that of the four quark condensate for $\kappa=1$ (see Eq. (8)). The solid line is the total contribution.

FIG. 2. The decay amplitude $F_{1}^{V}$ for the process $\Lambda_{c} \rightarrow \Lambda \ell \nu_{\ell}$ as function of the squared momentum transfer $t$ to the leptons. Solid line: Pole fit $F_{1}^{V}(t)=6.872 /(10.01-t)$ for $\kappa=2$ (see Eq.(8)) to the sum rule results (dots). Dashed line: the same for $\kappa=1 ; F_{1}^{V}(t)=2.393 /(4.716-t)$.

FIG. 3. The sum rule values for the decay amplitude $F_{1}^{V}$ at $t=0$ for the process $\Lambda_{b} \rightarrow \Lambda_{c} \ell \nu_{\ell}$ as function of the Borel mass $M_{F}^{2}$. The long-dashed line is the perturbative contribution, the short-dashed line that of the four quark condensate for $\kappa=1$ (see Eq. (8)). The solid line is the total contribution.

FIG. 4. The decay amplitude $F_{1}^{V}$ for the process $\Lambda_{b} \rightarrow \Lambda_{c} \ell \nu_{\ell}$ as function of the squared momentum transfer $t$ to the leptons. Solid line: Pole fit $F_{1}^{V}(t)=15.32 /(32.03-t)$ for $\kappa=2$ to the sum rule results (dots). Dashed line: the same for $\kappa=1 ; F_{1}^{V}(t)=8.12 /(22.27-t)$. 


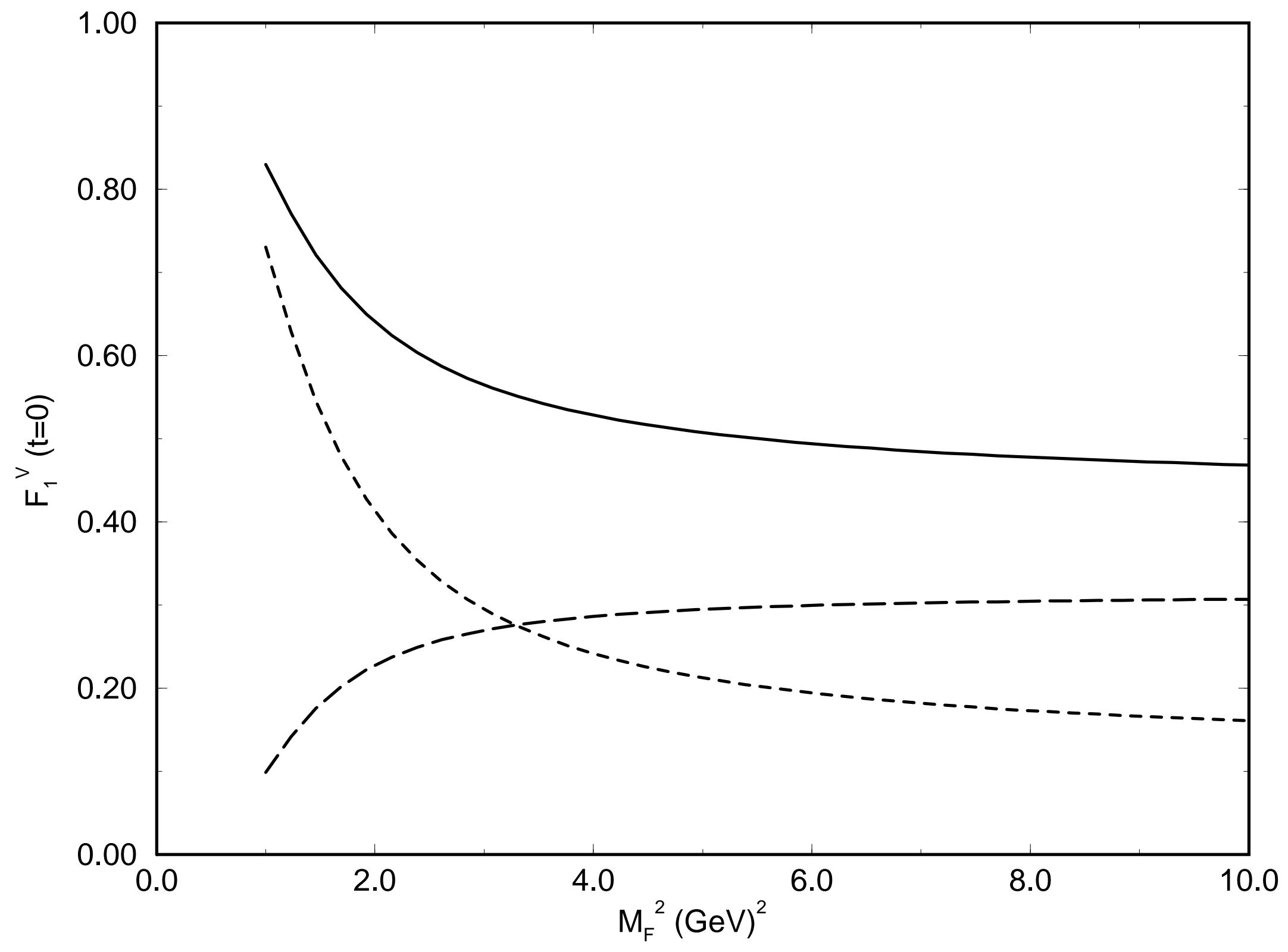




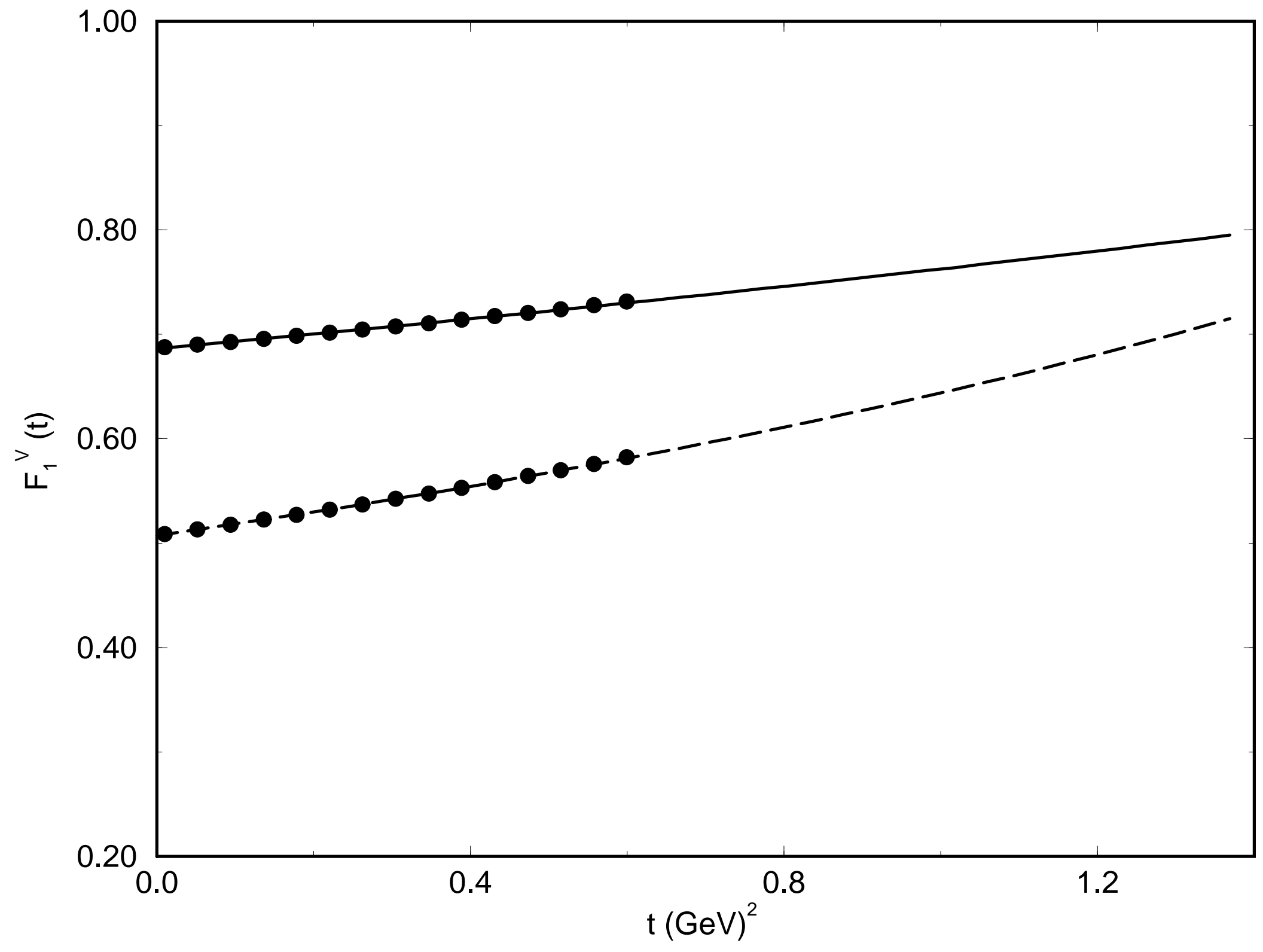




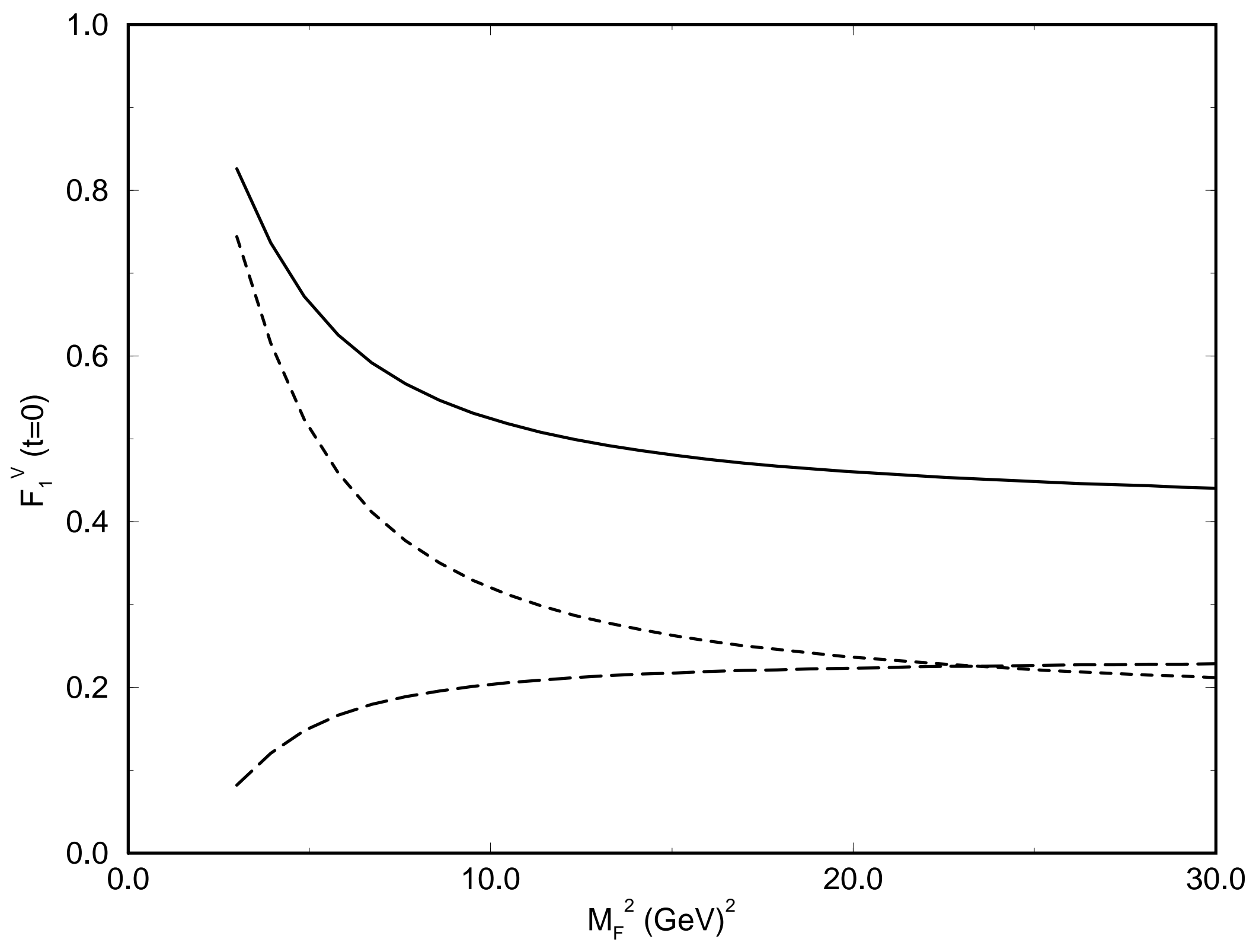




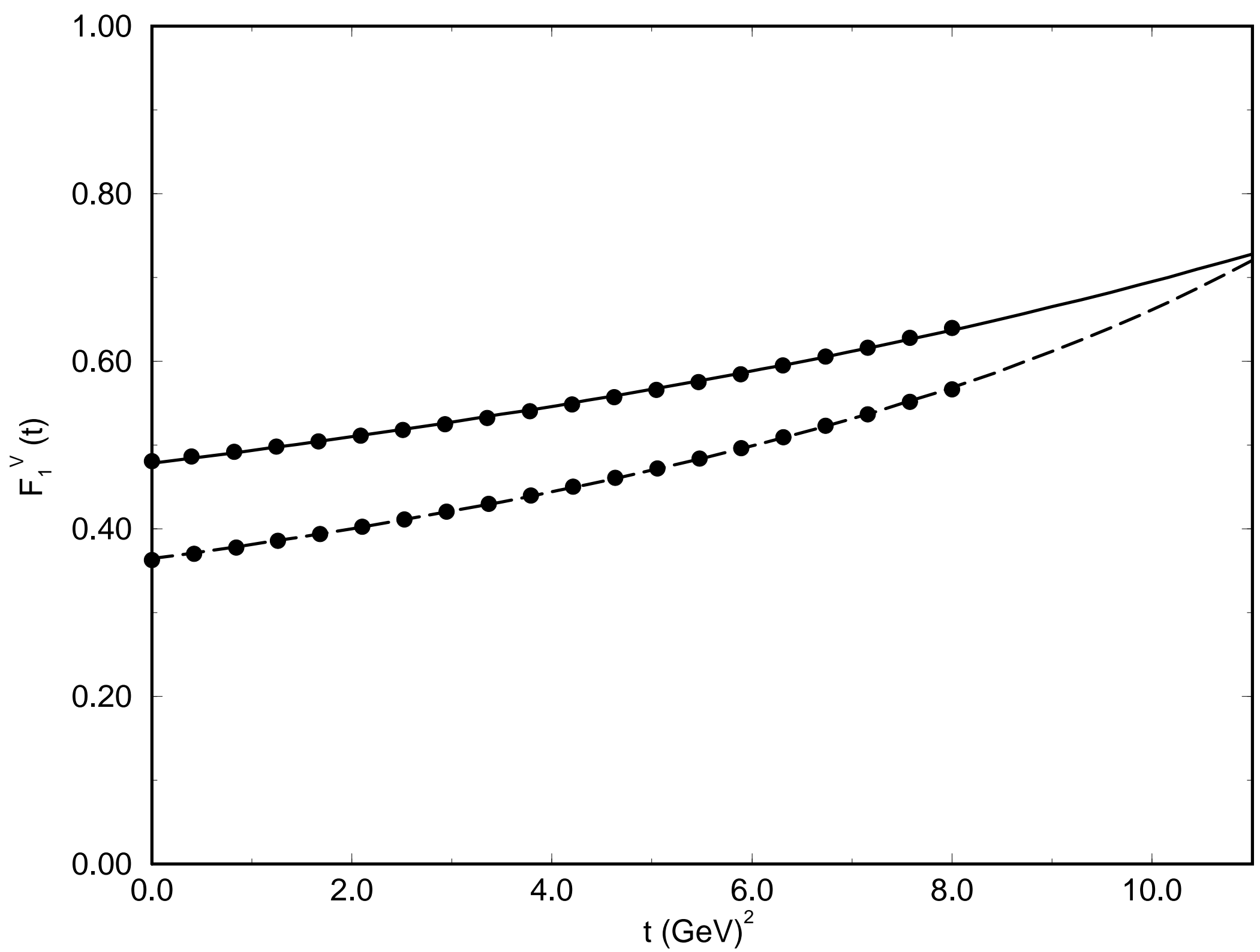

